\title{
Einfluss einer Mund-Nase-Maske auf die objektive körperliche Leistungsfähigkeit sowie das subjektive Belastungsempfinden bei gut-trainierten, gesunden Jungen
}

\author{
Benedikt Schulte-Körne (D) Wildor Hollmann · Argiris Vassiliadis · Hans-Georg Predel
}

Eingegangen: 16. Februar 2021 / Angenommen: 26. April 2021 / Online publiziert: 8. Juni 2021

(C) Der/die Autor(en) 2021

\begin{abstract}
Zusammenfassung Mit dieser Studie wurden die Effekte chirurgischer MNS auf die körperliche Leistungsfähigkeit sowie das subjektive Belastungsempfinden bei trainierten elfjährigen Jungen untersucht. Im aeroben und aerob-anaeroben Übergangsbereich fand sich keine Verminderung der objektiven Leistungsparameter. Allerdings zeigte sich auf der maximalen Belastungsstufe mit MNS eine signifikante Verminderung der Laufzeit sowie eine signifikante Erhöhung des subjektiven Belastungsempfindens bei ansonsten unveränderten Leistungsparametern. Zusammenfassend beeinflusst die Verwendung chirurgischer „Alltags“-MNS bei Kindern die sportlichen Aktivitäten nicht, solange die Belastungen primär im aeroben Intensitätsbereich durchgeführt werden.
\end{abstract}

Schlüsselwörter Kinder · MNS · Körperliche Leistungsfähigkeit · RPE

B. Schulte-Körne $(\bowtie) \cdot$ W. Hollmann · H.-G. Predel Institut für Kreislaufforschung und Sportmedizin, Deutsche Sporthochschule Köln, Am Sportpark Müngersdorf 6, 50933 Köln, Deutschland b.schulte@dshs-koeln.de

\section{W. Hollmann}

hollmann@dshs-koeln.de

H.-G. Predel

Predel@dshs-koeln.de

\section{A. Vassiliadis}

Olympiastützpunkt NRW/Rheinland,

Guts-Muths-Weg 1, 50933 Köln, Deutschland

argiris@osp-rheinland.de

\begin{abstract}
Effects of surgical face masks on exercise performance and perceived exertion of exercise in well-trained healthy boys
\end{abstract}

Summary This study investigated the effects of surgical "everday" face masks on the physical performance and perceived exertion in trained eleven-year-old boys.

No decrease in objective performance parameters was found in the aerobic and aerobic-anaerobic transition zones. However, at the maximum performance level with surgical face mask, there was a significant reduction in running time as well as a significant increase in the subjective perception of exertion with otherwise unchanged performance parameters. In summary, the use of surgical face masks in trained children does not affect athletic activities as long as the loads are performed primarily in the aerobic intensity range.

Keywords Children · Surgical mask · Exercise performance $\cdot$ RPE

\section{Hintergrund und Rationale}

Im Zuge der aktuellen SARS-Covid-2-Pandemie haben sich handelsübliche dreilagige "chirurgische“ Mund-Nase-Schutzmasken (MNS) - neben Abstand und Hygieneregeln - als ein Instrument zur Bekämpfung der Verbreitung von SARS-CoV-2 etabliert. Ihre konsequente Verwendung wird in vielen Berufs- und Alltagssituationen empfohlen bzw. verpflichtend vorgeschrieben [1]. Jedoch gibt es bzgl. der Verwendung von MNS im Rahmen sportlicher Aktivitäten, u.a. auch im Schulsport, bisher keine einheitlichen verbindlichen Empfehlungen [1]. Untersuchungen an Erwachsenen unter Verwendung von MNS zeigten keine signifikanten Effekte weder auf die körperliche Leistungsfähigkeit, Herzfrequenz (HF), Laktat, 
Tab. 1 Objektive und subjektive Leistungsparameter mit und ohne MNS auf dem Laufband

\begin{tabular}{|c|c|c|c|}
\hline & $\begin{array}{l}\text { Ohne MNS } \\
\text { (oMNS) }\end{array}$ & $\begin{array}{l}\text { Mit MNS } \\
\text { (mMNS) }\end{array}$ & $\begin{array}{l}\text { oMNS vs. mMNS } \\
\text { ( } p \text {-Wert) }\end{array}$ \\
\hline \multicolumn{4}{|c|}{ Höchste vollständig absolvierte Belastungsstufe } \\
\hline Herzfrequenz (bpm) & 189 & 191,4 & 0,36 \\
\hline Laktat (mmol/l) & 4,45 & 4,15 & 0,49 \\
\hline $\mathrm{SpO}_{2}(\%)$ & 97,2 & 97,8 & 0,17 \\
\hline Borg-Skala & 15,7 & 18,2 & $<0,01^{*}$ \\
\hline \multicolumn{4}{|c|}{ Laufgeschwindigkeit (km/h) } \\
\hline Bei $2,0 \mathrm{mmol} / \mathrm{l}$ Laktat & 11,7 & 11,7 & 0,96 \\
\hline Bei $4,0 \mathrm{mmol} / \mathrm{l}$ Laktat & 13,6 & 13,5 & 0,27 \\
\hline \multicolumn{4}{|l|}{ Laufzeit (s) } \\
\hline Gesamtlaufzeit (s) & 914,6 & 808,6 & $<0,01^{*}$ \\
\hline
\end{tabular}

Sauerstoffsättigung $\left(\mathrm{SpO}_{2}\right)$ noch auf das subjektive Belastungsempfinden (RPE) [2-7]. Jedoch zeigten sich negative Effekte auf die forcierte Vitalkapazität, den Peak Flow [2] und es wurde ein erhöhter Atemwegswiderstand beobachtet [7].

Bei Schulkindern hingegen finden sich bisher lediglich zwei Untersuchungen mit sog. „FFP2 (N95)“ -Masken, jedoch keine Studien zum Einfluss von „chirurgischen“ MNS [8]. Entsprechend liegen keine Daten zum Einfluss von MNS auf die körperliche/ sportliche Leistungsfähigkeit sowie das RPE bei Schulkindern vor.

Ziel dieser Studie war es daher, den Einfluss von MNS auf die maximale körperliche Leistungsfähigkeit, RPE sowie HF, Plasma-Laktatkonzentration und $\mathrm{SpO}_{2}$ bei 11-jährigen männlichen Fußballspielern zu untersuchen.

Auf der Grundlage der bisher verfügbaren Studien bei Erwachsenen wurde die Hypothese postuliert, dass bei Schulkindern keine Beeinträchtigung der objektiven sportlichen Leistungsfähigkeit durch das Tragen einer MNS auftritt.

\section{Probanden und Methoden}

\section{Probanden}

11 männliche gesunde, trainierte Spieler der U12-Fußball-Mannschaft des 1. FC Köln (11,3 $\pm 0,3$ Jahre, Größe: $149,8 \pm 5,8 \mathrm{~cm}$, Gewicht: 38,6 $44,4 \mathrm{~kg}$ ) wurden in die Studie einbezogen. Der Trainingsumfang beträgt zusätzlich zum Schulsport vier wöchentliche Trainingseinheiten.

\section{Studiendesign}

Prospektive, randomisierte, Cross-over-Studie.

\section{MNS (Mund-Nase-Schutzmaske)}

Dreilagige MNS der Firma Medi-Inn (Frechen, Germany, zertifiziert: EN 14683:2019 (Typ II)).

\section{Untersuchungen}

Für alle Probanden lag nach ausführlicher Information sowohl der Probanden als auch der Eltern eine schriftliche Einverständniserklärung der Eltern vor, weiterhin wurde die Studie von der lokalen Ethikkommission bewilligt. Jeder Proband absolvierte in randomisierter Reihenfolge auf dem Laufband (Firma H/P Cosmos sports \& medical GmbH (München, Deutschland)) zwei Untersuchungen (einmal mit MNS, einmal ohne MNS) in einem für diese Altersgruppe etablierten stufenförmigen Belastungsschema (Startgeschwindigkeit 2,4 m/s, Stufendauer $3,0 \mathrm{~min}$, Steigerung jeweils $0,4 \mathrm{~m} / \mathrm{s}$ ). Die beiden Belastungsuntersuchungen erfolgten in einem Abstand von exakt 7 Tagen, innerhalb derer die Probanden dem gewohnten Trainings- und Spielrhythmus nachgingen. Sämtliche Probanden wurden körperlich untersucht, die Körpertemperatur gemessen sowie der Peak Flow bestimmt. Alle Untersuchungen erfolgten unter Beachtung der aktuell gültigen Covid-19-Hygiene- und Infektionsschutzmaßnahmen.

Am Ende jeder Belastungsstufe wurden folgende Parameter ermittelt:

- Bestimmung der HF mittels Pulsgurt Polar T31 der Firma Polar Electro Europe AG (Steinhausen, Switzerland).

- RPE mittels standardisierter Borg-Skala (Gunnar Borg, 1982).

- Plasma-Laktatbestimmung mittels Abnahme von kapillärem Blut am Ohr (Auswertung: Biosen C-Line Gerät, EKFdiagnostic GmbH, Barleben, Germany) und Bestimmung der Laktatschwellen (2 und $4 \mathrm{mmol} / \mathrm{l}$ ) mit Hilfe des Computerprogrammes ,winlactat“ der Firma Mesics GmbH (Münster, Germany).

- Messung der $\mathrm{SpO}_{2}$ mittels Finger-Pulsoxymeter PO200 der Firma Pulox (Novidion GmbH, Köln, Germany).

\section{Statistische Analyse}

Die angegebenen Ergebnisse wurden als Mittelwerte \pm Standardabweichung dargestellt. Die weiteren statistischen Analysen (Shapiro-Wilk; Wilcoxon) wurden mit Hilfe von SPSS (IBM SPSS Statistics 27, Amonk, NY, USA) durchgeführt. Bei $p<0,05$ wurde eine Signifikanz angenommen.

\section{Ergebnisse}

In beiden Laufbanduntersuchungen (mit und ohne MNS) fanden sich bzgl. der Laufgeschwindigkeit $(\mathrm{km} / \mathrm{h})$ sowohl $\mathrm{im}$ aeroben $(2,0 \mathrm{mmol} / \mathrm{l}$ Laktat $)$ als auch im aerob-anaeroben $(4,0 \mathrm{mmol} / 1$ Laktat) Übergangsbereich keine signifikanten Unterschiede. Weiterhin zeigten sich auf allen Belastungsstufen für die Laktatkonzentration, die $\mathrm{HF}$ sowie die $\mathrm{SpO}_{2}$ mit und ohne MNS keine signifikanten Veränderungen 
Abb. 1 Subjektives Belastungsempfinden (RPE) bei den jeweiligen Belastungsstufen mit und ohne MNS

\section{RPE bei den jeweiligen Belastungsstufen mit und ohne MNS}

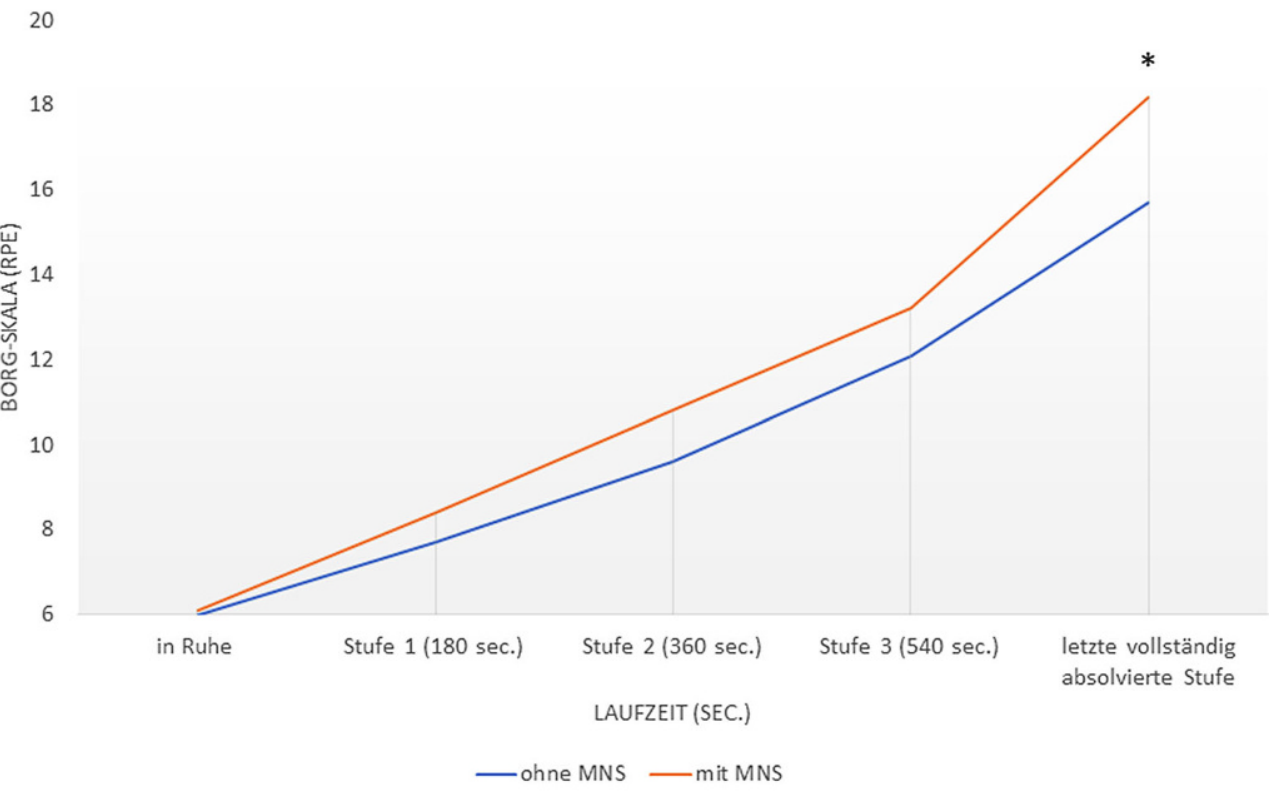

* $\mathrm{p}<0,01$ signifikant erhöht mit MNS

(Tab. 1). Im Gegensatz dazu konnte während der stufenförmigen Belastungsuntersuchungen mit MNS eine signifikante Verminderung der Gesamtlaufzeit (808,6 $\pm 142,2$ sec. vs. $914,6 \pm 131,0$ sec.; $p=0,001$, siehe Tab. 1) beobachtet werden. Auf der maximalen Belastungsstufe konnte zudem eine signifikante Erhöhung des subjektiven Belastungsempfindens (RPE: 18,2 vs. 15,7 BORG-Skala; $p=0,007$, siehe Abb. 1) mit MNS dokumentiert werden.

\section{Diskussion und Schlussfolgerung}

Angesichts der aktuellen Covid-19-Pandemie wird das Tragen von dreilagigen MNS (sog. „chirurgische Masken“) bei sportlichen Aktivitäten auch für Kinder, u.a. im Schulsport, diskutiert. Diese Diskussion wird bisher ohne das Vorliegen belastbarer wissenschaftlicher Untersuchungen zum Einfluss solcher MNS auf die körperliche/sportliche Leistungsfähigkeit von Kindern und Jugendlichen geführt. Mit dieser Studie wurden die Effekte dreilagiger MNS auf die körperliche Leistungsfähigkeit sowie das subjektive Belastungsempfinden im Rahmen einer stufenförmigen Laufbanduntersuchung bei elfjährigen gesunden Fußballern des 1. FC Köln evaluiert.

In dieser Untersuchung führte das Tragen einer MNS bei diesen gut-trainierten Jungen sowohl im aeroben ( $2 \mathrm{mmol} / \mathrm{l}$ Laktat) als auch im aerob-anaeroben (4 mmol/l Laktat) Übergangsbereich $\mathrm{zu}$ keiner Verminderung der objektiven Leistungsfähigkeit. Allerdings zeigte sich mit MNS im maximalen Belastungsbereich - entgegen unserer Ausgangshypothese - eine signifikante Verminderung der Gesamtlaufzeit und auf der maximalen Belastungsstufe eine signifikante
Erhöhung des subjektiven Belastungsempfindens. Angesichts fehlender signifikanter Unterschiede auf dieser maximalen Belastungsstufe hinsichtlich PlasmaLaktatkonzentration, $\mathrm{HF}$ und $\mathrm{SpO}_{2}$ kann für die Laufzeitverminderung auf Basis der vorliegenden Daten keine eindeutige Ursache benannt werden. Auffallend ist jedoch, dass sich erst unter maximaler Belastung ein erhöhtes subjektives Belastungsempfinden der Probanden mit MNS manifestiert, welches am ehesten durch einen erhöhten Atemwegswiderstand durch die chirurgische MNS [2, 7] erklärt werden kann. Weiterhin kann auch ein verändertes Atemmuster, welches nicht Gegenstand dieser Untersuchung war, hierzu beigetragen haben [7, 9]. Auch ein „NoceboEffekt“ kann nicht ausgeschlossen werden.

Limitationen dieser Studie stellen das kleine Untersuchungskollektiv sowie das Fehlen weiblicher Probanden dar.

Zusammenfassend legen diese Beobachtungen nahe, dass die Verwendung einer „chirurgischen Alltags“-MNS für Kinder aus physiologischer Sicht auch bei sportlichen Aktivitäten grundsätzlich möglich ist, insbesondere solange die Belastungen primär im aeroben Intensitätsbereich durchgeführt werden. Allerdings sollte berücksichtigt werden, dass die maximale Leistungsfähigkeit bei Kindern durch die MNS negativ beeinflusst werden kann. Künftige, weiterführende Studien sollten größere Probandenkollektive und unterschiedliche kindliche Alterskohorten inklusive weiblicher Probanden einbeziehen.

Funding Open Access funding enabled and organized by Projekt DEAL. 
Interessenkonflikt B. Schulte-Körne, W. Hollmann, A. Vassiliadis und H.-G. Predel geben an, dass kein Interessenkonflikt besteht.

Open Access Dieser Artikel wird unter der Creative Commons Namensnennung 4.0 International Lizenz veröffentlicht, welche die Nutzung, Vervielfältigung, Bearbeitung, Verbreitung und Wiedergabe in jeglichem Medium und Format erlaubt, sofern Sie den/die ursprünglichen Autor(en) und die Quelle ordnungsgemäß nennen, einen Link zur Creative Commons Lizenz beifügen und angeben, ob Änderungen vorgenommen wurden.

Die in diesem Artikel enthaltenen Bilder und sonstiges Drittmaterial unterliegen ebenfalls der genannten Creative Commons Lizenz, sofern sich aus der Abbildungslegende nichts anderes ergibt. Sofern das betreffende Material nicht unter der genannten Creative Commons Lizenz steht und die betreffende Handlung nicht nach gesetzlichen Vorschriften erlaubt ist, ist für die oben aufgeführten Weiterverwendungen des Materials die Einwilligung des jeweiligen Rechteinhabers einzuholen.

Weitere Details zur Lizenz entnehmen Sie bitte der Lizenzinformation auf http://creativecommons.org/licenses/by/4. 0/deed.de.

\section{Literatur}

1. Epidemiologisches Bulletin 19/2020 des RKI (7. Mai 2020), https://www.rki.de/DE/Content/Infekt/EpidBull/Archiv/ 2020/Ausgaben/19_20.pdf?_blob=publicationFile. Zugegriffen: 15.04.2021.

2. Fikenzer S, Uhe T, Lavall D, et al. Effects of surgical and FFP2/N95 face masks on cardiopulmonary exercise capaci- ty. Clin Res Cardiol. 2020;109(12):1522-30. https://doi.org/ 10.1007/s00392-020-01704-y.

3. Epstein D, Korytny A, Isenberg Y, et al. Return to training in the COVID-19 era: The physiological effects of face masks during exercise. Scand J Med Sci Sports. 2021;31(1):70-5. https:// doi.org/10.1111/sms.13832.

4. Roberge RJ, Kim JH, Benson SM. Absence of consequential changes in physiological, thermal and subjective responses from wearing a surgical mask. Respir Physiol Neurobiol. 2012;181(1):29-35. https://doi.org/10.1016/j.resp.2012.01. 010.

5. Hopkins SR, Dominelli PB, Davis CK, et al. Face masks and the cardiorespiratory response to physical activity in health and disease. Ann Am Thorac Soc. 2021;18(3):399-407. https://doi.org/10.1513/AnnalsATS.202008-990CME.

6. Shein SL, Whitticar S, Mascho KK, et al. The effects of wearing facemasks on oxygenation and ventilation at rest and during physical activity. Plos One. 2021;16(2):e247414. https://doi.org/10.1371/journal.pone.0247414.

7. Lässing J, Falz R, Pökel C, et al. Effects of surgical face masks on cardiopulmonary parameters during steady state exercise. Sci Rep. 2020;10(1):22363. https://doi.org/10.1038/ s41598-020-78643-1.

8. Eberhart M, Orthaber S, Kerbl R. The impact of face masks on children-A mini review. Acta Paediatr. 2021; https:// doi.org/10.1111/apa.15784.

9. Smith CL, Whitelaw JL, Davies B. Carbon dioxide rebreathing in respiratory protective devices: influence of speech and work rate in full-face masks. Ergonomics. 2013;56(5):781-90. https://doi.org/10.1080/00140139. 2013.777128.

Hinweis des Verlags Der Verlag bleibt in Hinblick auf geografische Zuordnungen und Gebietsbezeichnungen in veröffentlichten Karten und Institutsadressen neutral. 